\title{
Effectiveness of Multidetector Computed Tomography in Prosthetic Valve Endocarditis
}

\author{
Kayo Sugiyama, Hirotaka Watanuki, Masaho Okada, Yasuhiro Futamura, Masayuki Saito, \\ Satoshi Makino, Katsuhiko Matsuyama
}

Department of Cardiac Surgery, Aichi Medical University Hospital, Nagakute, Japan

Email: kayotaro3@gmail.com

How to cite this paper: Sugiyama, K., Watanuki, H., Okada, M., Futamura, Y., Saito, M., Makino, S. and Matsuyama, K. (2021) Effectiveness of Multidetector Computed Tomography in Prosthetic Valve Endocarditis. Open Journal of Thoracic Surgery, 11, 31-35.

https://doi.org/10.4236/ojts.2021.111005

Received: January 6, 2021

Accepted: March 15, 2021

Published: March 18, 2021

Copyright $\odot 2021$ by author(s) and Scientific Research Publishing Inc. This work is licensed under the Creative Commons Attribution International License (CC BY 4.0).

http://creativecommons.org/licenses/by/4.0/

\begin{abstract}
Background: Redo aortic valve replacement for prosthetic valve endocarditis is a challenge for surgeons. Echocardiography is occasionally not an effective modality for the detection of infectious signs in prosthetic valve endocarditis. Case presentation: Herein, we report the case of a patient whose prosthetic valve endocarditis was detected by multidetector computed tomography and who successfully underwent redo aortic valve replacement. Preoperative echocardiography revealed no remarkable findings related to endocarditis such as perivalvular leakage or vegetation; however, multidetector computed tomography revealed a thickened right coronary cusp. Intraoperatively, the right coronary cusp was confirmed to be covered with thick infected tissue. The pathological findings revealed broad destruction due to infection of the right coronary cusp. Conclusion: Multidetector computed tomography was useful in detecting infectious signs in prosthetic valves.
\end{abstract}

\section{Keywords}

Prosthetic Valve Endocarditis, Multidetector Computed Tomography, Compromised Host

\section{Introduction}

Echocardiography is a well-known, effective modality for diagnosing infective endocarditis (IE); however, it can occasionally be difficult to evaluate signs of infection in prosthetic valve endocarditis (PVE). Echocardiography is the "gold standard" for assessing the anatomy of cardiac valves and peri-valvular apparatus; however, the effectiveness may be limited by the patient's morphology and artifacts due to valvular calcifications or prosthetic material [1] [2]. Multidetector computed tomography (MDCT) may be effective for the structural evalua- 
tion of prosthetic valves because it can eliminate artifacts caused by artificial structures that are peculiarly seen on echocardiography [2]. By synchronizing the imaging acquisition with the cardiac pulsation, a more detailed image of the intracardiac structure can be obtained in this modality.

Herein, we report a case of a patient who successfully underwent redo aortic valve replacement after an accurate diagnosis of PVE by MDCT.

\section{Case Presentation}

The patient was a 64-year-old woman who had undergone ventricular septal defect closure at the age of 22. She underwent residual shunt closure and pulmonary valve repair for pulmonary valve stenosis at the age of 45 . One year previously, she was diagnosed with bicuspid aortic valve induced progression of aortic valve stenosis and ascending aortic dilatation and underwent the following procedures: 1) aortic valve replacement using a 23-mm INSPIRIS RESILIA aortic valve $^{\mathrm{TM}}$ (Edwards Lifesciences, Irvine, USA) for aortic valve stenosis of the bicuspid aortic valve; 2) graft replacement for dilated ascending aorta using a 30-mm Triplex one-branched graft ${ }^{\mathrm{TM}}$ (Terumo Corporation, Tokyo, Japan); and 3) left atrial appendage closure for chronic atrial fibrillation. She was also suffering from idiopathic pancytopenia, with laboratory data as follows: white blood cell count, $2.3 \times 10^{3} / \mu \mathrm{L}$; neutrophils, $56 \%$; hemoglobin level, $9.1 \mathrm{mg} / \mathrm{dL}$; platelet count, $5.0 \times 10^{5} / \mu \mathrm{L}$; C-reactive protein level, $0.04 \mathrm{mg} / \mathrm{dL}$; procalcitonin, $0.06 \mathrm{ng} / \mathrm{mL}$; and N-terminal pro-brain natriuretic peptide, $49 \mathrm{pg} / \mathrm{mL}$. Four months previously, she had developed a high fever due to sepsis. Antibiotic therapy was initiated because methicillin-resistant coagulase-negative staphylococci were detected in the blood culture. After antibiotic treatment with teicoplanin for 4 weeks, she was discharged with a prescription for oral tedizolid phosphate; however, it was discontinued because of severe nausea. Transthoracic and transesophageal echocardiography during hospitalization did not detect any significant signs related to PVE.

Four months later, she was readmitted in an emergency, complaining of fever and general fatigue. Transthoracic and transesophageal echocardiography did not detect any signs related to PVE (Figure 1(a)); however, MDCT revealed thickening of the right coronary cusp (Figure 1(b), Figure 1(c)). Preoperative laboratory data indicated inflammatory changes and pancytopenia, with the following results: white blood cell count, $2.5 \times 10^{3} / \mu \mathrm{L}$; neutrophils, $63 \%$; hemoglobin level, $10.5 \mathrm{mg} / \mathrm{dL}$; platelet count, $5.6 \times 10^{5} / \mu \mathrm{L}$; C-reactive protein level, 2.3 $\mathrm{mg} / \mathrm{dL}$; procalcitonin, $0.28 \mathrm{ng} / \mathrm{mL}$; and $\mathrm{N}$-terminal pro-brain natriuretic peptide, $187 \mathrm{pg} / \mathrm{mL}$.

Therefore, surgical treatment was performed through a median re-sternotomy. Cardiopulmonary bypass was instituted via bicaval drainage and ascending aortic cannulation. After cardiac arrest induced through antegrade cardioplegia, the exposure of the prosthetic valve was obtained through the ascending aorta. The right coronary cusp was covered with thick vegetation (Figure 2(a)); however, the remaining leaflets appeared intact, and there was no evidence of an obvious 


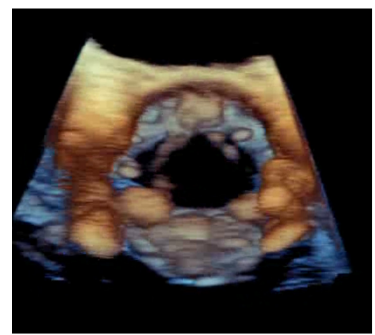

(a)

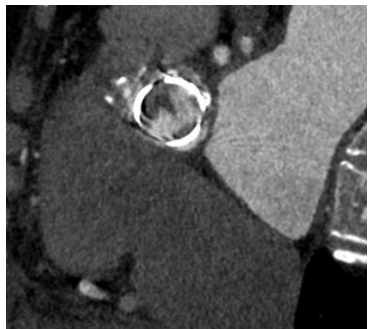

(b)

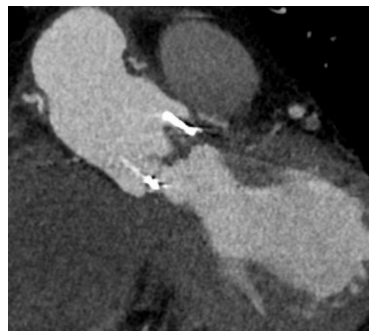

(c)

Figure 1. (a) Preoperative transesophageal echocardiography showing no remarkable infectious signs in the bioprosthesis; (b) Preoperative multidetector computed tomography showing thickening of the right coronary cusp in the bioprosthesis (axial view); (c) Preoperative multidetector computed tomography showing thickening of the right coronary cusp in the bioprosthesis (sagital view).

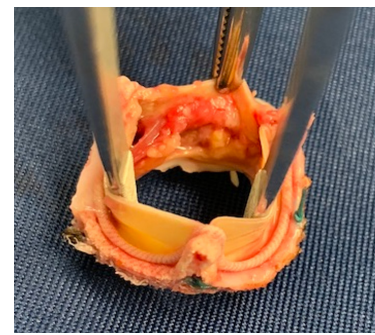

(a)

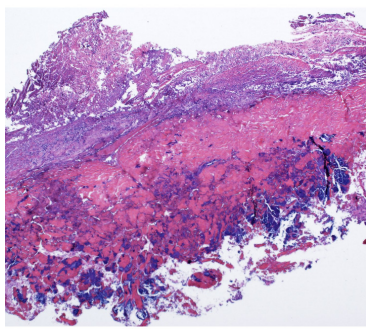

(b)

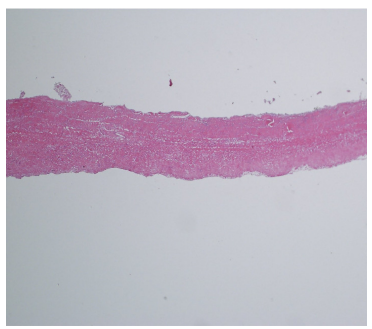

(c)

Figure 2. (a) Resected prosthetic valve with thickened vegetation in one leaflet without destruction of the annulus; (b) Postoperative pathological findings showing broad destruction with a thickened mass of bacteria and dense collection of neutrophils and macrophages in the leaflet; (c) Postoperative pathological findings showing an almost intact leaflet without any infectious signs.

annular abscess or detachment of the prosthetic valve. After complete removal of the previous prosthesis, redo aortic valve replacement with a 23-mm INSPIRIS RESILIA aortic valve ${ }^{\mathrm{TM}}$ (Edwards Lifesciences, Irvine, USA) was performed. The patient was weaned from cardiopulmonary bypass without difficulty, and the postoperative course was uneventful. As the intraoperative culture also showed the growth of methicillin-resistant coagulase-negative staphylococci, injections of tedizolid phosphate were initiated. After 4 weeks of intravenous tedizolid phosphate administration, oral intake of clindamycin was initiated at the time of discharge. Pathological findings revealed broad destruction due to infection in the right coronary cusp (Figure 2(b)); however, the remaining coronary cusps and prosthetic annulus were intact (Figure $2(\mathrm{c})$ ). The patient is currently recovering well and has not presented any symptoms related to the recurrence of infection for 12 months.

\section{Discussion}

This report presents a case with PVE in aortic valve bioprosthesis that has been successfully treated by surgery. Transthoracic and transesophageal echocardiography did not detect any significant findings related to infection, and MDCT 
was effective for the evaluation of prosthetic valve leaflets.

Although echocardiography is known to be an effective modality for diagnosing infective endocarditis, it sometimes cannot detect the signs of infection in PVE [1] [2]. The diagnosis of infectious endocarditis is based on the modified Duke criteria, as well as clinical, biological, and imaging findings. Despite being the "gold standard" for assessing cardiac valves and perivalvular apparatus, echocardiography may be ineffective due to the patient's morphology and artifacts caused by valvular calcifications or prosthetic material [1] [2]. Echocardiography has limited ability for perivalvular complications, especially for PVE [3]. Furthermore, echocardiography requires a highly trained operator, and the results are, to a certain degree, operator-dependent. For patients with high clinical suspicion of IE, multimodality imaging such as MDCT should be considered to confirm or rule out IE [3]. Although its relative limitations imposed by radiation exposure and the risk of nephrotoxicity associated with the injection of iodinated contrast [3], MDCT can effectively evaluate the structure of prosthetic valves by synchronizing the imaging acquisition with the cardiac pulsation to eliminate artifacts caused by artificial structure and provide a more detailed image of the intracardiac structure. MDCT has demonstrated promising results in valvular and perivalvular damage, providing high-resolution anatomic information and affording multiplanar reformations [2]. In accordance with Sollini et al., the " $3 \mathrm{M}$ " approach consisting of multimodality, multitracers, and multidisciplinary is essential for the detection of cardiovascular infections [4]. In the case of PVE, three other imaging-based findings are now included as either major or minor criteria to be considered: 1) cardiac computed tomography (CT);2) fluorodeoxyglucose (FDG) positron emission tomography (PET)/CT or white blood cell (WBC) single-photon emission CT (SPECT)/CT; and 3) embolic events or infectious aneurysms [4].

The present case revealed severe destruction due to infection in the right coronary cusp, intraoperatively and pathologically; however, there were no evident infectious signs in the annulus. There have been few reports describing the durability against infection of INSPIRIS RESILIA aortic valve. Shang et al. reported that the aggregation of macrophages and giant cells was reduced in an in vitro rabbit model with novel bovine pericardial tissue [5]. This study revealed a reduced inflammatory response in a novel bovine pericardial tissue treated with aldehyde capping chemistry and glycerolization [5]. Although further studies regarding durability are warranted, the INSPIRIS RESILIA aortic valve showed durability against infection in the annulus in the present case.

\section{Conclusion}

The patient successfully underwent redo aortic valve replacement for prosthetic valve endocarditis. While echocardiography did not detect infectious signs because of artifacts, MDCT was useful for detecting infectious signs in prosthetic valves. 


\section{Acknowledgements}

We would like to thank the Honyaku Center for reviewing and editing the manuscript and our colleagues for their insightful comments.

\section{Conflicts of Interest}

The authors declare no conflicts of interest regarding the publication of this paper.

\section{References}

[1] Habib, G., Lancellotti, P., Antunes, M.J., Bongiorni, M.G., Casalta, J.P., Del Zotti, F., et al. (2015) ESC Guidelines for the Management of Infective Endocarditis. European Heart Journal, 36, 3075-3123. https://doi.org/10.1093/eurheartj/ehv319

[2] Grob, A., Thuny, F., Villacampa, C., Flavian, A., Gaubert, J.Y., Raoult, D., et al. (2014) Cardiac Multidetector Computed Tomography in Infective Endocarditis: A Pictorial Essay. Insights Imaging, 5, 559-570. https://doi.org/10.1007/s13244-014-0353-1

[3] Erba, P.A., Pizzi, M.N., Roque, A., Salaun, E., Lancellotti, P., Tornos, P., et al. (2019) Multimodality Imaging in Infective Endocarditis. An Imaging Team within the Endocarditis Team. Circulation, 140, 1753-1765. https://doi.org/10.1161/CIRCULATIONAHA.119.040228

[4] Sollini, M., Berchiolli, R., Bolton, R.C.D., Rossi, A., Kirienko, M., Boni, R., et al. (2017) The "3M" Approach to Cardiovascular Infections: Multimodality, Multitracers, and Multidisciplinary. Seminars in Nuclear Medicine, 12, 199-224. https://doi.org/10.1053/j.semnuclmed.2017.12.003

[5] Shang, H., Claessens, S.M., Tian, B. and Wright, G.A. (2017) Aldehyde Reduction in a Novel Pericardial Tissue Reduces Calcification Using Rabbit Intramuscular Model. Journal of Materials Science: Materials in Medicine, 28, 16. https://doi.org/10.1007/s10856-016-5829-8 